\title{
Review on Green Synthesized Nanocomposites and Their Biological Activities
}

\author{
Sumit Ringwal $^{1}$ • Ankit Singh Bartwal ${ }^{1}$ - Aditya Ram Semwal ${ }^{2} \cdot$ Satish Chandra Sati $^{1 *}$ \\ ${ }^{1}$ Department of Chemistry, H.N.B. Garhwal University (A Central University) Srinagar Garhwal, Uttarakhand, \\ India-246174 \\ ${ }^{2}$ Department of Chemistry, DAV PG College Dehradun, Uttarakhand, India-248001
}

*Corresponding Author Email id: $\underline{\text { sati_2009@ rediffmail.com }}$

Received: 02.04.2021; Revised: 02.05.2021; Accepted: 15.05.2021

(C) Society for Himalayan Action Research and Development

\begin{abstract}
In this paper, we reviewed the present status of synthesis of nano structured materials for technological development as bimetallic, trimetallic and various organic, inorganic polymers nanocomposites in the field of nanosciences. Nanocomposites play an important role in the field of sciences, engineering and industries due to their high catalytic power, high optical, electrical and mechanical properties, which can be very useful in the field of biosensors, nano medicines and many more as social welfare factor. There are many techniques used for the preparation of nanocomposites. Among them, green method is commonly used technique for the synthesis of nanomaterials which is cost-effective, eco-friendly and less hazardous materials for the environment. Here we attempt to present an elaborate work done in the field of nanocomposites dwelling upon their advantages, challenges and future prospects.
\end{abstract}

Keywords: Nanocomposites $\bullet$ Bimetallic $\bullet$ Trimetallic $\bullet$ Green Method $\bullet$ Bioactivities

\section{Introduction}

Nowadays, researchers are taking an enormous interest in the field of inorganic as well as organic polymer nanocomposites due to their unexpected hybrid characteristics, which are synthesized by heterogeneous combinations of various components as basic reactants and among the composites layered structured nano composites have been studied extensively for last decades (Sanchez et.al., 2001; Usuki et.al., 1993). Nanocomposites exhibit improved properties namely medicinal strength, moduli, thermal stability and other properties when compared with pure polymers or conventional micro and macro size composites. The enhanced properties have been achieved by synthesis of nanoscale materials via various approaches. Nanocomposites are utilized to produce optically efficient materials.
Like if semiconductor nanoparticle is added with polymer, ceramic matrix materials or glass, there is enormous change in its optical property including absorption, fluorescence, and luminescence. In such kind of system, small size nanoparticles enhance optical properties while matrix material stabilized the particle size and growth (Burnside et al., 2000; Choi et.al., 2000; Byun et.al., 2001; Krikorian et.al., 2002; Xie et.al., 2002; Bhardwaj et.al., 2002).

Other applications of nanocomposite structures have resulted in transparent materials with unusually high RI, magnetic properties, and excellent mechanical properties. Nanocomposite structures provide a new method to improve the process ability and stability of materials with interesting optical properties. The applications of 
such composites are extremely broad, ranging from solid-state amplifier films to transparent magnets. This review focuses on recent developments in the synthesis and applications in the field of nanocomposite and nanotechnology.

\section{Green method}

This is one of the most widely applied methods for the synthesis of nanocomopsites because it is eco-friendly and it does not employ any toxic chemicals. Following steps are involved in green methods.

\section{(a) Preparation of Extracts}

Some amount of dried powdered plant material is addedto solvent in $500 \mathrm{~mL}$ round bottom flask and mixed well. The preparation of extracts is done by using magnetic heating stirrer at $70^{\circ} \mathrm{C}$ for $30 \mathrm{~min}$. The extracts obtained is centrifuged then filtered and filtrate is kept at refrigerator for further use further (Sati et.al., 2020a).

(b) Green synthesis of the Metal NPs by using plant extracts

In a typical synthesis of metal NPs, plant extracts is added to the metal salt or metal oxide solution (particular molarity) with desired ratio at $80^{\circ} \mathrm{C}$ with constant stirring. Reduction of metal ion take place around $3 \mathrm{~min}$, as monitored by UV-Vis technique. The color of the reaction mixtures gradually changes in $3 \mathrm{~min}$ at $80^{\circ} \mathrm{C}$ which indicate the formation of metal nanoparticles.The colored solution of metal NPs is then centrifuged till color completely disappear (Bartwalet.al., 2020; Sati et.al., 2020b).

(c) Synthesis of the bimetallic or trimetallic nanocomposites

For green synthesis of bimetallic or trimetallic nanocomposites some amount of metal saltor metal oxide is dispersed with few $\mathrm{mL}$ of plant extracts under continuous stirring. After $15 \mathrm{~min}$, few $\mathrm{mL}$ (in a fixed ratio with respect to plant extracts) of other metal oxide or metal salt is added to this mixture and stirred at $80^{\circ} \mathrm{C}$ for $4 \mathrm{~h}$. Finally, the prepared bimetallic or trimetallic nanocomposites as separated by a magnetic separator, is washed with suitable solvent and then dried at $90^{\circ} \mathrm{C}$ (Ayinde et.al., 2018; Atarod et.al., 2016; Azizi et.al., 2016; Atarod et.al., 2016).

Table1: Nanocomposites, Morphology and their activity

\begin{tabular}{|c|c|c|c|c|c|c|}
\hline $\begin{array}{c}\text { S. } \\
\text { No. }\end{array}$ & $\begin{array}{c}\text { Name of plant } \\
\text { (Common name) }\end{array}$ & $\begin{array}{l}\text { Part } \\
\text { Used }\end{array}$ & $\begin{array}{c}\text { Type of } \\
\text { NCs } / \\
\text { morphology }\end{array}$ & $\lambda_{\max }(\mathrm{in} \mathrm{nm})$ & $\begin{array}{c}\text { Characterization } \\
\text { techniques }\end{array}$ & Activity [Ref.] \\
\hline 1 & $\begin{array}{l}\text { Citrus paradise } \\
\text { (grape- } \\
\text { fruit red) }\end{array}$ & $\mathbf{P}$ & $\begin{array}{l}\text { Ag-MgO } \\
\text { spherically } \\
\text { dispersed }\end{array}$ & $\begin{array}{l}\text { AgNPs at } 440 \\
\text { NCsat } 380\end{array}$ & $\begin{array}{l}\text { UV ,TEM, XRD, } \\
\text { FTIR ,SEM, EDX }\end{array}$ & $\begin{array}{l}\text { Antibacterial } \\
\text { (Ayinde et.al., } \\
2018 \text { ) }\end{array}$ \\
\hline 2 & $\begin{array}{l}\text { Withania } \\
\text { coagulans } \\
\text { (Paneerphool) }\end{array}$ & $\mathbf{L}$ & $\begin{array}{l}\mathrm{Pd} / \mathrm{RGO} / \\
\mathrm{Fe}_{3} \mathrm{O}_{4}\end{array}$ & $\begin{array}{l}\text { PdNPsat } 263 \\
\text { NCsat } 270\end{array}$ & $\begin{array}{l}\text { XRD, FE-SEM, } \\
\text { EDS, UV, VSM, } \\
\text { TEM, FTIR, }\end{array}$ & $\begin{array}{l}\text { Catalytic activity } \\
\text { (Atarod et.al., } \\
2016 \text { ) }\end{array}$ \\
\hline 3 & wild ginger & EO & $\begin{array}{l}\text { ZnO-Ag } \\
\text { hexagonal } \\
\text { ZnO NPs }\end{array}$ & $\begin{array}{l}\text { AgNPs at } 430 \\
\text { NCs at } 352\end{array}$ & $\begin{array}{l}\text { UV, TEM, EDX, } \\
\text { XRD, FTIR. }\end{array}$ & $\begin{array}{l}\text { Antibacterial, } \\
\text { antimicrobial } \\
\text { (Azizi et.al., 2016) }\end{array}$ \\
\hline 4 & $\begin{array}{l}\text { Euphorbia } \\
\text { heterophylla } \\
\text { (Mexican) } \\
\text { fireplant }\end{array}$ & $\mathbf{L}$ & $\mathrm{Ag} / \mathrm{TiO}_{2}$ & $\begin{array}{l}\text { AgNPs at } \\
250-350\end{array}$ & $\begin{array}{l}\text { UV, XRD, } \\
\text { EDS,FESEM, FT-IR }\end{array}$ & $\begin{array}{l}\text { Catalytic activity } \\
\text { (Atarod et.al., } \\
\text { 2016) }\end{array}$ \\
\hline 5 & $\begin{array}{l}\text { Euphorbia } \\
\text { wallichii } \\
\text { (Wallich spurge) }\end{array}$ & $\mathbf{L}$ & $\begin{array}{l}\mathrm{Cu} / \mathbf{R G O} / \\
\mathrm{Fe}_{3} \mathrm{O}_{4} \\
\text { Spherical }\end{array}$ & $\begin{array}{l}\text { CuNPs at } 550 \\
\text { to } 580 \\
\text { NCs at } 265\end{array}$ & $\begin{array}{l}\text { FESEM, EDS, } \\
\text { TEM,BET,XRD, } \\
\text { FT-IR,elemental } \\
\text { mapping, VSM }\end{array}$ & $\begin{array}{l}\text { Catalytic activity } \\
\text { (Atarod et.al., } \\
\text { 2015) }\end{array}$ \\
\hline 6 & $\begin{array}{l}\text { Melissa } \\
\text { Officinalis L. }\end{array}$ & $\mathbf{L}$ & $\mathrm{CuO} / \mathrm{ZnO}$ & $\begin{array}{l}\text { CuONPs } \\
\text { At } 270\end{array}$ & $\begin{array}{l}\text { SEM, Elemental } \\
\text { mapping, EDS, TEM, }\end{array}$ & $\begin{array}{l}\text { Catalytic activity } \\
\text { (Bordbar et.al., }\end{array}$ \\
\hline
\end{tabular}


J. Mountain Res. P-ISSN: 0974-3030, E-ISSN: 2582-5011

Vol. 16(1), (2021), 181-186

DOI: https://doi.org/10.51220/jmr.v16il.18

\begin{tabular}{|c|c|c|c|c|c|c|}
\hline & (Lemon balm) & & & & XRD & 2018) \\
\hline 7 & $\begin{array}{l}\text { Euphorbia } \\
\text { neriifolia } \mathrm{L} \\
\text { (Indian Spurge) }\end{array}$ & $\mathbf{L}$ & Pd/perlit & $\begin{array}{l}\text { Extract shows } \\
\text { bands at } 368 \\
\text { and } 281\end{array}$ & $\begin{array}{l}\text { TEM,EDS,XRD, } \\
\text { FESEM,FT-IR }\end{array}$ & $\begin{array}{l}\text { Catalytic activity } \\
\text { (Maryami et.al., } \\
\text { 2017) }\end{array}$ \\
\hline 8 & $\begin{array}{l}\text { Ranunculus } \\
\text { muricatus. } \\
\text { rough-fruited } \\
\text { buttercup }\end{array}$ & $\mathbf{W P}$ & $\mathrm{Au} / \mathrm{TiO}_{2}$ & - & $\begin{array}{l}\text { XRD, SEM,TEM, } \\
\text { FT-IR }\end{array}$ & $\begin{array}{l}\text { Bacterial } \\
\text { inactivation (Tahir } \\
\text { et.al., 2016) }\end{array}$ \\
\hline 9 & $\begin{array}{l}\text { Acalypha } \\
\text { indica L. (Indian } \\
\text { Acalypha), }\end{array}$ & $\mathbf{L}$ & $\begin{array}{l}\mathrm{Cu} / \text { sodium } \\
\text { borosilicate }\end{array}$ & CuNPs at558 & $\begin{array}{l}\text { SEM, EDS, TEM, } \\
\text { XRD, BET,FT-IR }\end{array}$ & $\begin{array}{l}\text { Catalytic activity } \\
\text { (Nasrollahzadeh } \\
\text { et.al., 2018) }\end{array}$ \\
\hline 10 & $\begin{array}{l}\text { Cuscuta reflexa } \\
\text { (Giant dodder) }\end{array}$ & $\mathbf{L}$ & $\begin{array}{l}\mathrm{Cu} / \mathrm{GO} / \\
\mathrm{MnO}_{2}\end{array}$ & CuNPs at 575 & $\begin{array}{l}\text { XRD, FESEM, BET, } \\
\text { TGA, VSM, EDS, } \\
\text { FT-IR }\end{array}$ & $\begin{array}{l}\text { Catalytic activity } \\
\text { (Naghdi et.al., } \\
\text { 2018) }\end{array}$ \\
\hline 11 & $\begin{array}{l}\text { Salvadora } \\
\text { persica } \mathrm{L} \text {. } \\
\text { (Mustard tree) }\end{array}$ & $\mathbf{R E}$ & $\begin{array}{l}\text { Pd@ } \\
\text { graphene } \\
\text { Pd NPs } \\
\text { was }(f c c)\end{array}$ & $\begin{array}{l}\text { GRO at } 230, \\
301, \mathrm{PdCl}_{2} \text { at } \\
420, \mathrm{SP}-\mathrm{HRG}- \\
\text { Pd- at } 1280\end{array}$ & $\begin{array}{l}\text { UV, XRD, TEM, FT- } \\
\text { IR, XPS Raman }\end{array}$ & $\begin{array}{l}\text { Catalytic activity } \\
\text { (Al-Marri et.al., } \\
\text { 2016) }\end{array}$ \\
\hline 12 & $\begin{array}{l}\text { Citrus paradisi. } \\
\text { (Grapefruit) }\end{array}$ & $\mathbf{F}$ & $\begin{array}{l}\text { Silk-AuNPs } \\
\text { quasi- } \\
\text { spherical, } \\
\text { hexagonal, and } \\
\text { triangle shapes }\end{array}$ & $\begin{array}{l}\text { AuNPs at } \\
540\end{array}$ & $\begin{array}{l}\text { DRS, SEM, TEM, } \\
\text { LSCM }\end{array}$ & $\begin{array}{l}\text { Unique optical } \\
\text { properties } \\
\text { (Nolasco et.al., } \\
2013 \text { ) }\end{array}$ \\
\hline 13 & $\begin{array}{l}\text { Euphorbia } \\
\text { peplus Linn }\end{array}$ & $\mathbf{L}$ & $\begin{array}{l}\mathbf{A g} / \mathrm{Fe}_{3} \mathrm{O}_{4} \\
\mathrm{Ag} / \mathrm{Fe}_{3} \mathrm{O}_{4} \\
\text { spherical }\end{array}$ & AgNPs at450 & $\begin{array}{l}\text { XRD, TEM, EDS, } \\
\text { FT-IR, FE-SEM }\end{array}$ & $\begin{array}{l}\text { Catalytic activity } \\
\text { (Sajjadi et.al., } \\
\text { 2017) }\end{array}$ \\
\hline 14 & $\begin{array}{l}\text { Mortiño } \\
\text { Vaccinium } \\
\text { floribundum } \\
\text { (Kunth) }\end{array}$ & B & Ag-Graphene & $\begin{array}{l}\text { broad peak in } \\
\text { between } 240- \\
340 \text { and } 480- \\
530\end{array}$ & $\begin{array}{l}\text { FT-IR. UV, XRD, } \\
\text { SEM, TEM }\end{array}$ & $\begin{array}{l}\text { Photo catalytic } \\
\text { activities (Vizuete } \\
\text { et.al., 2016) }\end{array}$ \\
\hline 15 & $\begin{array}{l}\text { Mentha longifolia } \\
\text { (horse mint) }\end{array}$ & $\mathbf{L}$ & $\begin{array}{l}\text { ZnO and } \\
\text { ZnO/CuO } \\
\text { Spherically - } \\
\text { distributed } \\
\text { particles }\end{array}$ & $\begin{array}{l}\mathrm{ZnO}(\mathrm{W}) \text { at } \\
370 \\
\mathrm{ZnO}(\text { Ext }) \text { at } \\
370\end{array}$ & $\begin{array}{l}\text { XRD, } \\
\text { EDX,SEM,TGA, } \\
\text { TEM, FT-IR, UV, } \\
\text { DRS, BET }\end{array}$ & $\begin{array}{l}\text { Anti-bacterial } \\
\text { activity } \\
\text { (Mohammadi } \\
\text { et.al., 2018) }\end{array}$ \\
\hline 16 & $\begin{array}{l}\text { Cylindrocladium } \\
\text { floridanum }\end{array}$ & $\mathbf{F}$ & $\begin{array}{l}\text { Nanogold- } \\
\text { Bio-composite } \\
\text { Spherical }\end{array}$ & AuNPs at 540 & $\begin{array}{l}\text { UV-Vis XRD, SEM, } \\
\text { EDX, TEM }\end{array}$ & $\begin{array}{l}\text { Heterogeneous } \\
\text { catalyst (Narayanan } \\
\text { et.al., 2011) }\end{array}$ \\
\hline 17 & $\begin{array}{l}\text { Euphorbia } \\
\text { helioscopia L } \\
\text { (sun spurge) }\end{array}$ & $\mathbf{L}$ & $\begin{array}{l}\mathbf{A g} / \mathbf{R G O} / \\
\mathbf{T i O}_{2} \\
\text { Analogous } \\
\text { structure to } \\
\mathrm{TiO}_{2}\end{array}$ & $\begin{array}{l}\mathrm{GO} / \mathrm{TiO}_{2} \\
\text { visible region } \\
\text { blue shift is } \\
\text { observed in } \\
\mathrm{TiO}_{2}\end{array}$ & $\begin{array}{l}\text { UV, TEM, XRD, } \\
\text { SEM, EDS, ICP,FT- } \\
\text { IR }\end{array}$ & $\begin{array}{l}\text { Catalytic activity } \\
\text { (Nasrollahzadeh } \\
\text { et.al., 2016) }\end{array}$ \\
\hline 18 & $\begin{array}{l}\text { Orchis mascula } L \text {. } \\
\text { (early spring } \\
\text { orchis) }\end{array}$ & $\mathbf{L}$ & $\begin{array}{l}\mathrm{Cu} / \text { egg } \\
\text { shell, } \mathrm{Fe}_{3} \mathrm{O}_{4} / \\
\text { eggshell } \\
\text { spherical } \\
\text { shaped (size 5- } \\
15 \mathrm{~nm} \text { ) }\end{array}$ & CuNPs at575 & $\begin{array}{l}\text { UV, DTA-TGA,FT- } \\
\text { IR, FE-SEM, EDS, } \\
\text { XRD, BET, VSM }\end{array}$ & $\begin{array}{l}\text { Catalytic activity } \\
\text { (Nasrollahzadeh } \\
\text { et.al., 2016) }\end{array}$ \\
\hline 19 & $\begin{array}{l}\text { Pulicaria } \\
\text { glutinosa }\end{array}$ & & $\begin{array}{l}\text { Graphene/ } \\
\text { Ag } \\
\text { AgNPs seems } \\
\text { FCC }\end{array}$ & $\begin{array}{l}\text { GRO at } 230 \\
\text { and } 301 \text {, } \\
\text { AgNPs at } 420\end{array}$ & UV, XRD, EDX & $\begin{array}{l}\text { Substrates for } \\
\text { SERS activities } \\
\text { (Al-Marri et.al., } \\
\text { 2015) }\end{array}$ \\
\hline
\end{tabular}




\begin{tabular}{|c|c|c|c|c|c|c|}
\hline 20 & $\begin{array}{l}\text { Lycopersicon } \\
\text { esculentum } \\
\text { (Tomato) }\end{array}$ & $\bar{F}$ & $\begin{array}{l}\text { Biocidal } \\
\text { Silver- } \\
\text { Activated } \\
\text { Charcoal } \\
\text { exfoliated } \\
\text { structure } \\
\text { almost- } \\
\text { transparent } \\
\text { single layer } \\
\text { GO }\end{array}$ & $\begin{array}{l}\text { AgNPs at } 410 \\
\text { NCs at } 410\end{array}$ & XRD, SEM, UV & $\begin{array}{l}\text { antimicrobial } \\
\text { activity, water } \\
\text { purification } \\
\text { (Arputha et.al., } \\
\text { 2013) }\end{array}$ \\
\hline
\end{tabular}

Abbreviation (NPs - Nanoparticles; NCs - Nanocomposites; P- Peels; EO- Essential Oil; L- Leaf; WPWhole Plant; B- Berries, RE - Root Extract; F- Fungus; RGO- Reduce Grapheme Oxide, FCC- Face Centered Cubic Cell)

\section{Conclusion}

Nanocomposites are one of the most important tools in the field of science, engineering, and industry also. Nanocomposites are one step advance than metallic nanoparticles because nanocomposites are like a junction between two or more nanoparticles and they have highly versatile property when compared to nanoparticles. Nanocomposites are very useful for sunlight-induced degradation of organic pollutants and wastewater treatment. Although a variety of photo catalysts have been designed toward this goal, various methods have been used in formation of bimetallic or trimetallic and various organic and inorganic polymers nanocomposites. Most of these methods are still in progressing stage.In this review paper it is concluded that nanocomposites synthesized by green method are excellent in differentbiological activitieswith high catalytic power.

\section{References}

Al-Marri AH, Khan M, Khan M, Adil SF, AlWarthan A, Alkhathlan HZ, ... \&Tahir, MN (2015). Pulicariaglutinosa extract: a toolbox to synthesize highly reduced graphene oxidesilver nanocomposites; Inte. J Mol. Sci., 16(1): 1131-1142.
Al-Marri AH, Khan M, Shaik MR, Mohri N, Adil SF, Kuniyil M, ... \&Siddiqui MRH (2016). Green synthesis of Pd@ grapheme nanocomposite: catalyst for the selective oxidation of alcohols; Arab. J Chem; 9(6): 835-845.

Arputha KVS, Dakshinamurthy A \& Selvakumar $\operatorname{PM}(2013)$. Eco-friendly biocidal silveractivated charcoal nanocomposite: antimicrobial application in water purification; Synth. React. Inor. Metatl-org. NanoMet. Chem. 43(8): 1068-1072.

Atarod M, Nasrollahzadeh M \& Sajadi SM (2015). Green synthesis of a Cu/reduced graphene oxide $/ \mathrm{Fe}_{3} \mathrm{O}_{4}$ nanocomposite using Euphorbia wallichii leaf extract and its application as a recyclable and heterogeneous catalyst for the reduction of 4-nitrophenol and rhodamine $B ; R S C$ advances; 5(111): 91532-91543.

Atarod M, Nasrollahzadeh M \& Sajadi SM (2016).Euphorbia heterophylla leaf extract mediated green synthesis of $\mathrm{Ag} / \mathrm{TiO}_{2}$ nanocomposite and investigation of its excellent catalytic activity for reduction of variety of dyes in water; $J$ Coll. Inter. Matri. 462: 272-279.

Atarod M, Nasrollahzadeh M \& Sajadi SM (2016). Green synthesis of $\mathrm{Pd} / \mathrm{RGO} / \mathrm{Fe}_{3} \mathrm{O}_{4}$ nanocomposite using Withania coagulans leaf extract and its application as 
magnetically separable and reusable catalyst for the reduction of 4-nitrophenol; $J$ coll. Inter. Sci., 465: 249-258.

Ayinde WB, Gitari MW, Muchindu M \& Samie A (2018). Biosynthesis of ultrasonically modified $\mathrm{Ag}-\mathrm{MgO}$ nanocomposite and its potential for antimicrobial activity; $J$ Nanotech; 2018.

Azizi S, Mohamad R, Rahim RA, Moghaddam $\mathrm{AB}$, Moniri M, Ariff A, ... \& Namvab $\mathrm{F}$ (2016). ZnO-Ag core shell nanocomposite formed by green method using essential oil of wild ginger and their bactericidal and cytotoxic effects; Appl. Surf. Sci.; 384: 517524.

Bartwal AS, Sumit\& Sati SC (2020). Biosynthesis of silver nanoparticles from flowers of Rhododenderon campanulatumtree of Tungnath Himalaya; Appl.Innov. Res., 2: 3943.

Bharadwaj RK, Mehrabi AR, Hamilton C, Trujillo C, Murga M, Fan R, ...\& Thompson AK (2002). Structure-property relationships in cross-linked polyester-clay nano composites; Polymer; 43(13): 3699-3705.

Bordbar M, Negahdar N \& Nasrollahzadeh M (2018). Melissa Officinalis L. leaf extract assisted green synthesis of $\mathrm{CuO} / \mathrm{ZnO}$ nanocomposite for the reduction of 4nitrophenol and Rhodamine B; Sep. Puri. Tech., 191: 295-300.

Burnside SD \& Giannelis EP (2000). J. Polymer Sci. Part B: Polym. Physics; 38: 1595.

Byun HY, Choi MH \& Chung IJ (2001). Synthesis and characterization of resol type phenolic resin/ layered silicate nanocomposites; Chem. Mater., 13(11): 42214226.

Choi MH, Chung IJ \& Lee JD (2000). Morphology and curing behaviors of phenolic resin-layered silicate nanocomposites prepared by melt intercalation; Chem. Mater.; 12(10): $2977-$ 2983.

Krikorian V, Kurian M, Galvin ME, Nowak AP, Deming TJ \&Pochan, DJ (2002). Polypeptide- based nanocomposite: Structure and properties of poly (L-lysine)/Na+montmorillonite; $J$ Polymer Sci. Part B: Polymer Physics; 40(22): 2579-2586.

Maryami M, Nasrollahzadeh M \&Sajadi SM (2017). Green synthesis of the Pd/perlite nanocomposite using Euphorbia neriifolia $L$. leaf extract and evaluation of its catalytic activity; Sep. Pur. Tech.; 184: 298-307.

Mohammadi AR, Habibi YA, Bayrami A, Latifi NS \& Asadi A (2018). Green synthesis of $\mathrm{ZnO}$ and $\mathrm{ZnO} / \mathrm{CuO}$ nanocomposites in Mentha longifolia leaf extract: characterization and their application as antibacterial agents; J Mater. Sci Matr. Electro., ; 29(16): 13596-13605.

Naghdi S, Sajjadi M, Nasrollahzadeh M, Rhee KY, Sajadi SM \& Jaleh B (2018). Cuscuta reflexa leaf extract mediated green synthesis of the $\mathrm{Cu}$ nanoparticles on graphene oxide/manganese dioxide nanocomposite and its catalytic activity toward reduction of nitroarenes and organic dyes; J Taiwan Inst. Chem. Eng.; 86: 158-173.

Narayanan KB \& Sakthivel N (2011). Synthesis and characterization of nano-gold composite using Cylindrocladium floridanum and its heterogeneous catalysis in the degradation of 4-nitrophenol; J Haza. Mater., 189(1-2): 519-525.

Nasrollahzadeh M, Atarod M, Jaleh B \& Gandomirouzbahani M (2016). In situ green synthesis of $\mathrm{Ag}$ nanoparticles on graphene oxide/TiO2 nanocomposite and their catalytic activity for the reduction of 4nitrophenol, congo red and methylene blue; Ceramics International; 42(7): 85878596. 
Nasrollahzadeh M, Sajadi SM \& Hatamifard A (2016). Waste chicken eggshell as a natural valuable resource and environmentally benign support for biosynthesis of catalytically active $\mathrm{Cu} /$ eggshell, $\mathrm{Fe}_{3} \mathrm{O}_{4} /$ eggshell and $\mathrm{Cu} / \mathrm{Fe}_{3} \mathrm{O}_{4} /$ eggshell nanocomposites; Appl.Catal. B: Env; 191: 209-227.

Nasrollahzadeh M, Sajjadi M, Dasmeh HR \& Sajadi SM (2018). Green synthesis of the $\mathrm{Cu} /$ sodium borosilicate nanocomposite and investigation of its catalytic activity; J Alloys Comp.; 763: 1024-1034.

Nolasco AV, Morales LR, Sánchez MV, Hinestroza JP, Castro LE \& Vilchis NAR (2013). Formation of silk-gold nanocomposite fabric using grapefruit aqueous extract; Textile Res. J; 83(12): 12291235.

Sajjadi M, Nasrollahzadeh M \&Sajadi SM (2017). Green synthesis of $\mathrm{Ag} / \mathrm{Fe}_{3} \mathrm{O}_{4}$ nanocomposite using Euphorbia peplus Linn leaf extract and evaluation of its catalytic activity: $J$ Coll. Inter. Sci., 497: 1-13.

Sanchez C, Soler-Illia GDA, Ribot F, Lalot T, Mayer CR \& Cabuil V (2001). Designed hybrid organic-inorganic nanocomposites from functional nanobuilding blocks; Chem. of Mater.; 13(10): 3061-3083.

Sati SC, Kour G, Bartwal AS \& Sati MD (2020a). Biosynthesis of Metal Nanoparticles from Leaves of Ficus palmata and Evaluation of Their Anti-inflammatory and Anti-diabetic Activities; Biochem.; 59(33): 3019-3025.

Sati SC, Sumit, Bartwal AS \&Agarwal AK (2020b). Green synthesis of silver nanoparticles from Citrus medica peels and determination of its antioxidant activity; Appl. Innov. Res., 2: 56-60.

Tahir K, Ahmad A, Li B, Khan AU, Nazir S, Khan S \& Khan SU (2016). Preparation, characterization and an efficient photocatalytic activity of
$\mathrm{Au} / \mathrm{TiO}_{2}$ nanocomposite prepared by green deposition method; Mater. Letters; 178: 5659.

Usuki A, Kawasumi M, Kojima Y, Okada A, Kurauchi T \& Kamigaito O (1993). Swelling behavior of montmorillonite cation exchanged for $\omega$-amino acids by caprolactam; J Mater. Res., 8(5): 1174-1178.

Vizuete KS, Kumar B, Vaca AV, Debut A \& Cumbal L (2016). Mortiño (Vaccinium floribundum Kunth) berry assisted green synthesis and photocatalytic performance of Silver-Graphene nanocomposite; J Photochem. Photobio. Chem., 329: 273-279.

Xie W, Xie R, Pan WP, Hunter D, Koene B, Tan LS \& Vaia R (2002). Thermal stability of quaternary phosphonium modified montmorillonites; Chem. Mater.,; 14(11): 4837-4845.

$* * * * * * *$ 